OPEN ACCESS

Edited by:

Giovanna Suzzi,

University of Teramo, Italy

Reviewed by:

Antonio Valero,

Universidad de Córdoba, Spain

Evgeny A. Semchenko,

Griffith University, Australia

Charles Lawrence Larson,

Rocky Mountain Laboratory,

United States

*Correspondence:

Byeonghwa Jeon

bjeon@ualberta.ca

Specialty section:

This article was submitted to

Food Microbiology

a section of the journal

Frontiers in Microbiology

Received: 16 December 2016

Accepted: 12 May 2017

Published: 30 May 2017

Citation:

Oh E, McMullen LM, Chui L and Jeon B (2017) Differential Survival of Hyper-Aerotolerant Campylobacter jejuni under Different Gas Conditions.

Front. Microbiol. 8:954. doi: 10.3389/fmicb.2017.00954

\section{Differential Survival of Hyper-Aerotolerant Campylobacter jejuni under Different Gas Conditions}

\author{
Euna Oh${ }^{1}$, Lynn M. McMullen², Linda Chui ${ }^{3,4}$ and Byeonghwa Jeon ${ }^{1 *}$ \\ 1 School of Public Health, University of Alberta, Edmonton, AB, Canada, ${ }^{2}$ Department of Agricultural, Food and Nutritional \\ Science, University of Alberta, Edmonton, AB, Canada, ${ }^{3}$ Department of Laboratory Medicine and Pathology, University of \\ Alberta, Edmonton, AB, Canada, ${ }^{4}$ Provincial Laboratory for Public Health, Edmonton, AB, Canada
}

Campylobacter jejuni accounts for a significant number of foodborne illnesses around the world. C. jejuni is microaerophilic and typically does not survive efficiently in oxygenrich conditions. We recently reported that hyper-aerotolerant (HAT) C. jejuni are highly prevalent in retail poultry meat. To assess the capabilities of HAT C. jejuni in foodborne transmission and infection, in this study, we investigated the prevalence of virulence genes in HAT C. jejuni and the survival in poultry meat in atmosphere at a refrigeration temperature. When we examined the prevalence of eight virulence genes in $70 \mathrm{C}$. jejuni strains from raw poultry meat, interestingly, the frequencies of detecting virulence genes were significantly higher in HAT C. jejuni strains than aerosenstive $C$. jejuni strains. This suggests that HAT C. jejuni would potentially be more pathogenic than aerosensitive C. jejuni. Under aerobic conditions, aerosensitive $C$. jejuni survived at $4^{\circ} \mathrm{C}$ in raw poultry meat for 3 days, whereas HAT C. jejuni survived in poultry meat for a substantially extended time; there was a five-log CFU reduction over 2 weeks. In addition, we measured the effect of other gas conditions, including $\mathrm{N}_{2}$ and $\mathrm{CO}_{2}$, on the viability of HAT C. jejuni in comparison with aerosensitive and aerotolerant strains. $\mathrm{N}_{2}$ marginally affected the viability of $\mathrm{C}$. jejuni. However, $\mathrm{CO}_{2}$ significantly reduced the viability of C. jejuni both in culture media and poultry meat. Based on the results, modified atmosphere packaging using $\mathrm{CO}_{2}$ may help us to control poultry contamination with HAT C. jejuni.

Keywords: Campylobacter, aerotolerance, virulence genes, bacterial survival, pathogen inhibition

\section{INTRODUCTION}

Campylobacter is a leading bacterial cause of human gastroenteritis, annually accounting for approximately 166 million diarrheal cases around the world, particularly in developed countries (Kirk et al., 2015). Campylobacter infection in humans develop fever, vomiting, abdominal pains, and diarrhea, and in some cases Guillain-Barré syndrome, an autoimmune disorder characterized by acute and progressive neuromuscular paralysis (Young et al., 2007). Human infection with C. jejun $i$ is facilitated by the function of various virulence factors involved in toxin production (e.g., $c d t A B C$ ), cell adhesion (e.g., $c a d F$, peb1A, and pldA) and invasion (e.g., ciaB), and colonization of gastrointestinal tracts (Bolton, 2015). 
Campylobacter is isolated from a wide range of domestic animals and wildlife (Jokinen et al., 2011). In particular, the gastrointestinal tracts of poultry are colonized by Campylobacter jejuni, the major human pathogenic species of Campylobacter, at the level of $10^{6} \sim 10^{8} \mathrm{CFU} / \mathrm{g}$ feces or higher (Hermans et al., 2011). Poultry meat is often contaminated with C. jejuni during poultry processing, and human campylobacteriosis is most frequently associated with the consumption of contaminated poultry products (Skarp et al., 2016). In addition, crosscontamination in the kitchen is also an important risk factor transferring Campylobacter (Chai et al., 2008; Luber, 2009). It has been estimated that a two-log reduction in the number of Campylobacter on chicken carcasses may lead to approximately a 30-fold reduction in the number of human campylobacteriosis cases (Rosenquist et al., 2003). To control Campylobacter contamination of poultry, various intervention strategies have been examined at the pre- and post-harvest levels, such as bacteriocin and bacteriophages (Hermans et al., 2011; Umaraw et al., 2017).

Unlike other enteric pathogenic bacteria, C. jejuni exhibits unique microbiological features. For example, C. jejuni is asaccharolytic and has limitations in the utilization of hexose sugars, including glucose, because of the lack of 6phosphofructokinase in the glycolysis pathway (Parkhill et al., 2000; Velayudhan and Kelly, 2002). To supply carbon sources, C. jejuni relies on the utilization of amino acids, organic acids (e.g., lactic acid), and fucose in some strains (Leach et al., 1997; Thomas et al., 2011; Stahl et al., 2012). In addition, C. jejuni is microaerophilic and capnophilic and requires both $\mathrm{O}_{2}$ and $\mathrm{CO}_{2}$ for growth preferably at $5-10 \%$ and $1-10 \%$, respectively (Bolton and Coates, 1983). Despite the fastidious nature of Campylobacter, it has not been understood how Campylobacter causes such a significant number of human infection cases around the world.

Various tolerance mechanisms have been reported to support the survival of Campylobacter under harsh stress conditions, such as heat, cold, acid, and desiccation stresses (Murphy et al., 2006). In addition, Campylobacter produces biofilms and switches its physiological state to a viable but nonculturable (VBNC) cell to promote survival under stress conditions. In C. jejuni, biofilm formation is stimulated under aerobic conditions, and aeration triggers the formation of VBNC cells (Oh et al., 2015b, 2016), suggesting C. jejuni is equipped with multiple survival mechanisms that may support the viability of C. jejuni under oxygen-rich conditions. Besides these survival mechanisms, aerotolerance would be the front-line survival mechanism of C. jejuni when this microaerophilic pathogen encounters the aerobic environment (Bronowski et al., 2014). Despite our perception about oxygen-sensitivity in C. jejuni, interestingly, we recently reported that hyper-aerotolerant (HAT) strains of $C$. jejuni are highly prevalent in retail poultry meat; the HAT strains survive longer than $24 \mathrm{~h}$ in vigorous aerobic shaking at $200 \mathrm{rpm}$. Also, HAT C. jejuni often belongs to the multilocus sequence typing (MLST) clonal complexes (CCs) that are frequently implicated in human infection (Oh et al., 2015a), suggesting that HAT C. jejuni might be closely related to human infection. To evaluate the virulence potential of
HAT C. jejuni, in this study, we investigated the prevalence of virulence genes in HAT C. jejuni strains. In addition, we measured the survival of HAT $C$. jejuni under different gas conditions, such as $\mathrm{N}_{2}$ and $\mathrm{CO}_{2}$, aiming to develop intervention strategies to control HAT $C$. jejuni in poultry meat by using modified atmosphere packaging (MAP) with different gases, since aerotolerance confers tolerance to oxygen, not other gases.

\section{MATERIALS AND METHODS}

\section{Bacterial Strains and Culture Conditions}

Seventy C. jejuni strains that were isolated from poultry were used in this study (Oh et al., 2015a). C. jejuni NCTC 11168 is the first genome-sequenced strain of Campylobacter and was used as a control in the study (Parkhill et al., 2000). C. jejuni 81176 was used as a positive control for PCR detection of virB11 (Bacon et al., 2002). In our previous study, we first reported high prevalence of HAT C. jejuni that can effectively survive in a vigorous aerobic condition, such as aerobic shaking at $200 \mathrm{rpm}$ (Oh et al., 2015a). Based on the level of aerotolerance, we arbitrarily divided C. jejuni into three different groups: (1) aerosensitive $C$. jejuni that loses viability before $12 \mathrm{~h}$ by aerobic shaking at $200 \mathrm{rpm},(2)$ aerotolerant C. jejuni that loses viability between $12 \sim 24 \mathrm{~h}$ by aerobic shaking at $200 \mathrm{rpm}$, and (3) HAT C. jejuni that survives even after $24 \mathrm{~h}$ of aerobic shaking at $200 \mathrm{rpm}$ (Oh et al., 2015a). The 70 C. jejuni poultry strains were isolated from retail poultry meat in our previous study and consisted of 20 aerosensitive strains, 25 aerotolerant strains, and 25 HAT strains (Oh et al., 2015a). The C. jejuni strains were routinely grown on Mueller-Hinton $(\mathrm{MH})$ agar plates (Difco) at $42^{\circ} \mathrm{C}$ under microaerobic conditions $\left(85 \% \mathrm{~N}_{2}, 5 \% \mathrm{O}_{2}\right.$ and $10 \% \mathrm{CO}_{2}$ ).

\section{Determination of $C$. jejuni Survival under Different Gas Conditions}

Campylobacter jejuni survival was determined in $\mathrm{MH}$ media and chicken meat at $4^{\circ} \mathrm{C}$ in normal atmospheric conditions and under $\mathrm{CO}_{2}$ and $\mathrm{N}_{2}$. Frozen C. jejuni strains in $10 \%$ glycerol were inoculated on $\mathrm{MH}$ agar plates and inoculated plates were incubated at $42^{\circ} \mathrm{C}$ under microaerobic condition. Overnight cultures of strains of $\mathrm{C}$. jejuni grown on $\mathrm{MH}$ agar plates were harvested with fresh $\mathrm{MH}$ broth and diluted in $\mathrm{MH}$ broth to an optical density at $600 \mathrm{~nm}\left(\mathrm{OD}_{600}\right)$ of 0.1 . The bacterial suspension was transferred to multiple 96-well plates, and the 96-well plates were incubated at $4^{\circ} \mathrm{C}$ in air and in an anaerobic jar filled with either $\mathrm{CO}_{2}$ or $\mathrm{N}_{2}$. In addition, $\mathrm{N}_{2}$ gas condition was constructed with $100 \%$ nitrogen gas flushing and $\mathrm{CO}_{2}$ condition was generated with gas pack $\left(>97 \% \mathrm{CO}_{2}\right)$. To prevent desiccation, a container with water was placed nearby the 96well plates in a refrigerator. Samples were taken at predetermined time for enumeration. In addition, the survival of two strains of C. jejuni, which were randomly chosen from each aerotolerance group [HAT strains (\#12 and \#21), aerotolerant strains (\#4 and \#29), and aerosensitive strains (\#24 and \#66)], was determined in raw chicken meat; these strains were selected from different 
MLST CCs based on their aerotolerance level. Approximately one-gram of raw chicken meat, including skin and muscle, was prepared with a sterilized razor and placed in a 12-well plate. After applying an aliquot $(100 \mu \mathrm{l})$ of $C$. jejuni suspension (approximately $8 \times 10^{8} \mathrm{CFU} / \mathrm{ml}$ ) onto each portion of meat and skin mixture, the plate was stored at $4^{\circ} \mathrm{C}$ under three different gas conditions, including normal atmosphere, $\mathrm{CO}_{2}$, and $\mathrm{N}_{2}$. Due to the potential indigenous $C$. jejuni in poultry meat, controls were prepared without addition of $C$. jejuni. The poultry meat samples were transferred to a $50 \mathrm{ml}$ tube containing $2 \mathrm{ml}$ of fresh $\mathrm{MH}$ broth. After vortexing for $2 \mathrm{~min}$, the supernatant was collected, serially diluted, and spread onto $\mathrm{MH}$ agar plates for enumeration. Each experiment was carried out with duplicate samples, and the experiment was repeated three times.

\section{PCR Detection of Virulence Genes}

Overnight cultures on $\mathrm{MH}$ agar at $42^{\circ} \mathrm{C}$ under microaerobic conditions of C. jejuni strains were collected in PBS ( $\mathrm{pH} 7.2)$. Bacterial suspension of overnight culture of $C$. jejuni strains were diluted in PBS to an OD600 of 0.01 (approximately, $8 \times 10^{6} \mathrm{CFU} / \mathrm{ml}$ ) and boiled for $10 \mathrm{~min}$ to release gDNA. After centrifugation, the supernatant was used as a template. To evaluate the potential virulence of HAT C. jejuni strains, we investigated the prevalence of eight important virulence genes (cadF, $c d t B, c i a B, \operatorname{doc} A, i a m$, peb1A, pldA, and virB11), which are associated with toxin production, cell adhesion and invasion, and colonization of gastrointestinal tracts in chickens with PCR with ExTaq polymerase (Takara, Japan). Primers used are listed in Table 1. The positive controls for six virulence genes, such as $c a d F, c d t B, c i a B, d o c A$, iam, peb1A and pldA, were amplified from C. jejuni NCTC11168, and virB11 was amplified from C. jejuni 81-176. The PCR mixture was amplified with the following conditions: initial denaturation at $96^{\circ} \mathrm{C}$ for $3 \mathrm{~min}$ followed by 35 cycles of denaturation $96^{\circ} \mathrm{C}$ for $30 \mathrm{~s}$, variable annealing temperature $\left(c d t B, c i a B, c a d F\right.$ and $p l d A$ at $45^{\circ} \mathrm{C}, d o c A, p e b 1$ and virB11 at $50^{\circ} \mathrm{C}$, iam at $53^{\circ} \mathrm{C}$ ) for $30 \mathrm{~s}$, extension at $72^{\circ} \mathrm{C}$ for $1 \mathrm{~min}$
$20 \mathrm{~s}$ and the final extension at $72^{\circ} \mathrm{C}$ for $7 \mathrm{~min}$. The results were analyzed by electrophoresis with $1 \%$ agarose gels and SYBR safe staining dye (Invitrogen).

\section{Statistical Analysis}

Two-way ANOVA was performed by using GraphPad Prism 6 (GraphPad Software Inc., United States). Chi-square distribution was used to analyze if the prevalence of virulence genes is dependent on aerotolerance by using SPSS Statistics 21.0 (IBM Predictive Software, United States).

\section{RESULTS}

\section{Effect of Aerotolerance on C. jejuni Survival in Chicken Meat}

To evaluate the impact of hyper-aerotolerance on the survival of C. jejuni in poultry meat in this study, raw poultry meat was spiked with two strains of $C$. jejuni from each aerotolerance group (i.e., aerosenstive, aerotolerant, and HAT C. jejuni groups) and incubated at $4^{\circ} \mathrm{C}$ under aerobic conditions. The aerosensitive C. jejuni strains lost their viability on poultry meat within 3 days, and the aerotolerant $C$. jejuni strains survived for 7 days (Figure 1). Interestingly, HAT C. jejuni strains survived in poultry meat for 2 weeks (Figure 1). This means that HAT C. jejuni strains survived in food in atmospheric conditions approximately four times longer than aerosensitive strains of C. jejuni. The results showed that aerotolerance significantly affects the viability of $C$. jejuni in poultry meat under aerobic conditions.

\section{Prevalence of Virulence Genes in HAT C. jejuni Strains}

In 70 strains of $C$. jejuni from poultry meat, the frequencies of detecting virulence genes were 100, 97.1, 68.6, 81.4, 57.1, 84.3, 64.3 , and $11.4 \%$ for $c a d F, c d t B, c i a B$, docA, iam, peb1, pldA, and

TABLE 1 | Primers used in this study.

\begin{tabular}{|c|c|c|c|c|}
\hline Gene & Primer & Sequence $\left(5^{\prime}-3^{\prime}\right)$ & Size (bp) & Reference \\
\hline \multirow[t]{2}{*}{ cadF } & cadF_F & TTGAAGGTAATITAGATATG & 400 & Konkel et al., 1999a \\
\hline & cadF_R & CTAATACCTAAAGTTGAAAC & & \\
\hline \multirow[t]{2}{*}{$c d t B$} & cdtB_F & GTTAAAATCCCCTGCTATCAACCA & 495 & Bang et al., 2001 \\
\hline & cdtB_R & GTTGGCACTTGGAATITGCAAGGC & & \\
\hline \multirow[t]{2}{*}{$\operatorname{ciaB}$} & ciaB_F & GTTAAAGTTGGCAGT & 1163 & Konkel et al., 1999a \\
\hline & ciaB_R & GTTCTITAAATाПTCATAATGC & & \\
\hline \multirow[t]{2}{*}{ docA } & docA_F & ATAAGGTGCGGTITGGC & 725 & Muller et al., 2006 \\
\hline & docA_R & GTCTITGCAGTAGATATG & & \\
\hline \multirow[t]{2}{*}{ iam } & iamA_F & GCACAAAATATATCATTACAA & 518 & Konkel et al., 1999a \\
\hline & iamA_R & TTCACGACTACTATGAGG & & \\
\hline \multirow[t]{2}{*}{ peb1 } & peb1_F & TAATACGACTCACTATAGGGGAAAATCTाT & 775 & Biswas et al., 2011 \\
\hline & peb1_R & ПTाTCGCTAAAGCATCAATTCATT & & \\
\hline \multirow[t]{2}{*}{ pldA } & pldA_F & AAGCTTATGCGTIIT & 913 & Datta et al., 2003 \\
\hline & pldA_R & TATAAGGCTITCTCCA & & \\
\hline \multirow[t]{2}{*}{ virB11 } & virB11_F & GAACAGGAAGTGGAAAAACTAGC & 708 & Bacon et al., 2002 \\
\hline & virB11_R & TTCCGCATTGGGCTATATG & & \\
\hline
\end{tabular}




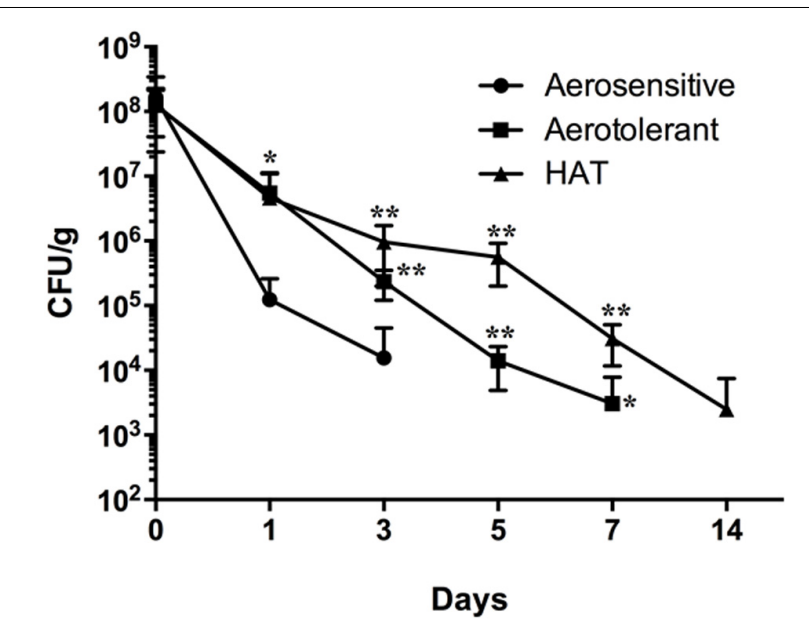

FIGURE 1 | Survival of aerosensitive, aerotolerant, and HAT C. jejuni strains in poultry meat at $4^{\circ} \mathrm{C}$ under aerobic conditions. Two $C$. jejuni strains were randomly selected from each aerotolerance group and used to spike raw poultry meat in duplicate. The results indicate the means and standard deviations of duplicate samples of the two different strains in a single experiment. Three independent experiments were performed, and similar results were obtained in all the experiments. The statistical analysis was performed with two-way ANOVA in comparison with aerosensitive strains. ${ }^{*} P \leq 0.05,{ }^{* *} P \leq 0.01$.

virB11, respectively (Figure 2 and Table 2). When we clustered the results based on the aerotolerance level, interestingly, the detection frequencies in HAT C. jejuni strains were higher than those in aerosensitive C. jejuni strains (Table 2), suggesting that HAT C. jejuni would potentially be more pathogenic to humans than aerosensitive C. jejuni.

\section{Viability of HAT C. jejuni Strains in Different Gas Atmospheres}

The survival of HAT C. jejuni measured under different gaseous conditions. In the food industry, MAP is often employed to extend the microbial shelf-life of meat, and $\mathrm{O}_{2}, \mathrm{~N}_{2}$, and $\mathrm{CO}_{2}$ are the major gases used for MAP. Thus, we selected $\mathrm{N}_{2}$ and $\mathrm{CO}_{2}$ for the viability testing of HAT C. jejuni strains. Consistent with their aerotolerance level, there was about approximately a four $\log$ reduction in CFU in aerosensitive strains of C. jejuni, a three $\log \mathrm{CFU}$ reduction in aerotolerant strains, and a two $\log \mathrm{CFU}$ reduction in HAT strains of $\mathrm{C}$. jejuni at $4^{\circ} \mathrm{C}$ in $\mathrm{MH}$ broth under the normal atmospheric conditions within 3 days. Incubation in $\mathrm{N}_{2}$ reduced the survival of $C$. jejuni, and $\mathrm{CO}_{2}$ further decreased CFU counts in HAT C. jejuni, compared with the aerobic conditions (Figure 3 ). The CFU reduction in all the strains were similar between days 3 and 7 (Figure 3), and no C. jejuni was detected in day 14 (data not shown).

\section{Impact of Different Gas Atmosphere on the Survival of HAT C. jejuni in Poultry Meat}

The viability of C. jejuni strains belonging to different aerotolerance groups was determined in poultry meat stored in different gas atmospheres. In $\mathrm{N}_{2}$, aerotolerant and HAT C. jejuni strains were detected for 14 days, whereas aerosensitive strains survived for 7 days (Figure 4A). Compared to aerobic conditions (Figure 1), $\mathrm{N}_{2}$ did not reduce the viability of HAT C. jejuni strains in poultry meat. In $\mathrm{CO}_{2}$, however, HAT strains of C. jejuni survived only for a week (Figure 4B); this is a significant viability reduction compared to atmospheric conditions where HAT C. jejuni strains survived for 2 weeks in poultry meat (Figure 1). The results exhibit that HAT C. jejuni did not survive well in $\mathrm{CO}_{2}$, compared to aerobic conditions.

\section{DISCUSSION}

Despite the well-known microaerophilic characteristic of C. jejuni, our previous study showed that some C. jejuni strains are highly tolerant to aerobic stress and these strains are highly prevalent in poultry meat (Oh et al., 2015a). In addition, Rodrigues et al. (2015) recently characterized an unique human isolate of $C$. jejuni strain, named $\mathrm{Bf}$, which can grow aerobically, suggesting that some $C$. jejuni strains are highly resistant to aerobic stress. Increased tolerance to aerobic stress would enable C. jejuni to survive during transmission to humans through foods. This would significantly impact the safety of poultry meat because of frequent contamination of poultry meat by Campylobacter. In this study, we demonstrated that HAT C. jejuni survived in raw poultry meat at $4^{\circ} \mathrm{C}$ significantly longer than aerosensitive C. jejuni (Figure 1), confirming the potential threat of HAT C. jejuni on the safety of fresh poultry meat.

The $c a d F$ and $c d t$ genes are detected in C. jejuni strains from poultry at high frequencies (Rozynek et al., 2005). Similarly, in this study, $c a d F$ and $c d t$ genes were detected in all and most (97.1\%) C. jejuni strains, respectively (Table 2). The iam locus has been detected in $C$. jejuni chicken isolates at 54.7\% (Rozynek et al., 2005). The pldA and ciaB genes have been detected from C. jejuni poultry isolates at the frequencies of 63.6 and $67.3 \%$, respectively (Melo et al., 2013). Hanning et al. (2010) reported relatively low detection frequencies of ciaB (40\%) and pldA $(56 \%)$ in C. jejuni isolates from poultry carcasses. The virB11 gene is located in the virulence plasmid pVir, which is often detected in C. jejuni strains that cause bloody diarrhea (Bacon et al., 2002; Tracz et al., 2005). The prevalence of virB11 was $10.7 \sim 17 \%$ in human clinical isolates and $9.5 \sim 14 \%$ in poultry isolates (Datta et al., 2003; Tracz et al., 2005). When the results were sorted based on the aerotolerance level, the frequencies of detecting virulence genes were significantly higher in HAT C. jejuni strains in comparison with aerosenstive C. jejuni strains (Figure 2 and Table 2). Interestingly, the most substantial differences in the frequency of detection were observed in the genes associated with invasion, including $c i a B$ and $i a m$ (Figure 2 and Table 2). CiaB shares similarities with SipB (Salmonella invasion protein B) from Salmonella and IpaB (invasion plasmid antigen B) from Shigella flexneri and is translocated to human epithelial cells. Even though a knockout mutation of ciaB does not affect C. jejuni adhesion to INT407 cells, it significantly 

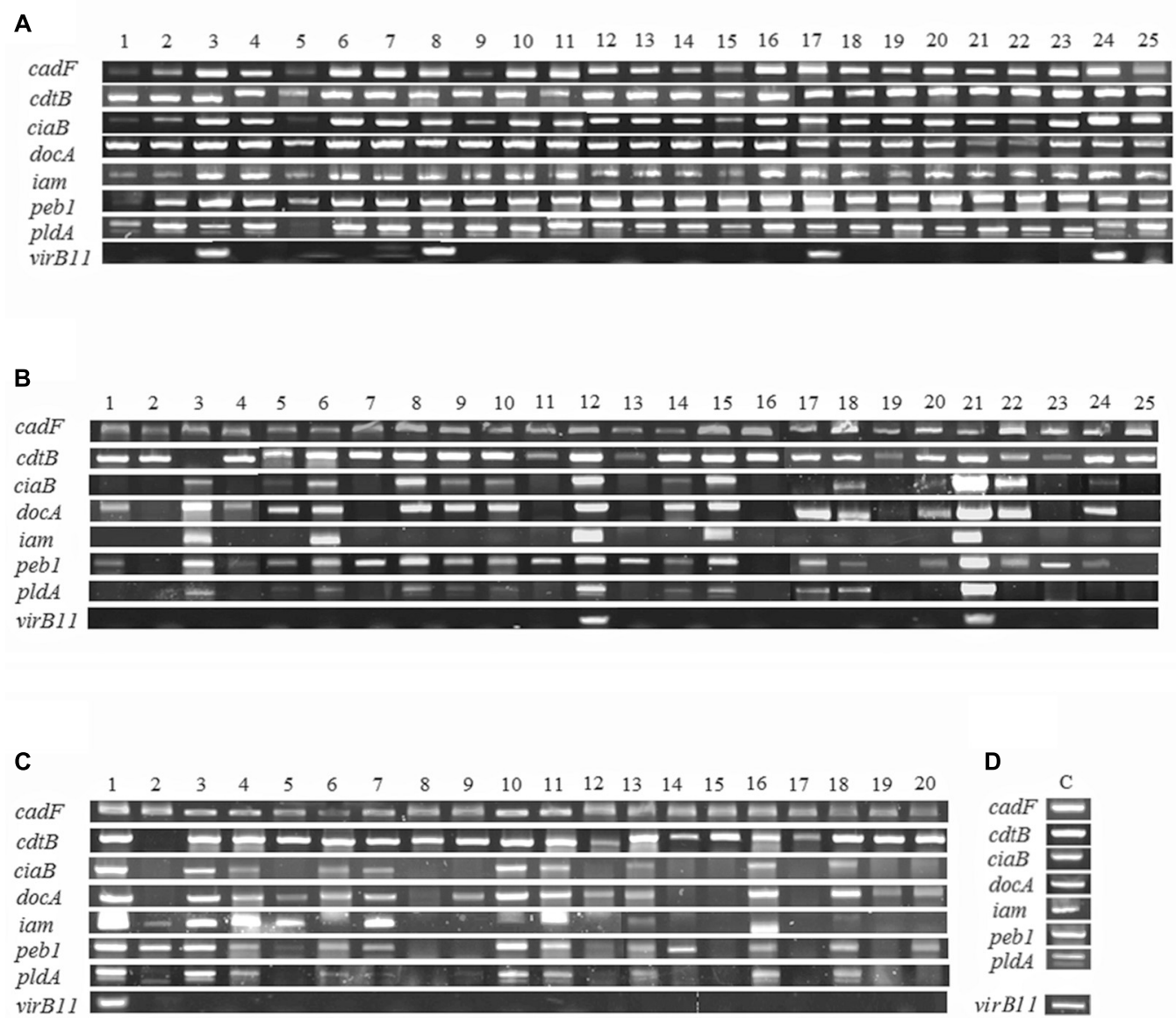

FIGURE 2 | Detection of virulence genes in 70 strains of $C$. jejuni from poultry meat. The results show the prevalence of eight virulence genes in hyper-aerotolerant (A), aerotolerant (B), and aerosensitive (C) strains of C. jejuni. Positive controls (D) were amplified from C. jejuni NCTC11168 (cadF, cdtB, ciaB, docA, iam, peb1 and pldA) and 81-176 (virB11). Controls were included each batch of PCR testing, and representative results were presented.

TABLE 2 | Prevalence (\%) of virulence genes in 70 isolates of $C$. jejuni from poultry meat.

\begin{tabular}{|c|c|c|c|c|c|c|c|c|}
\hline & $\operatorname{cadF}^{\mathrm{ND}}$ & $c d t B^{N S}$ & $\operatorname{ciaB}^{* * * *}$ & $\operatorname{doc} A^{* *}$ & $\operatorname{iam}^{* * * *}$ & peb1* & $p l d A^{* * * *}$ & $\operatorname{virB11}{ }^{\mathrm{NS}}$ \\
\hline HAT C. jejuni $(n=25)$ & 100 & 100 & 100 & 100 & 100 & 100 & 96 & 20 \\
\hline Aerotolerant C. jejuni $(n=25)$ & 100 & 96 & 52 & 68 & 20 & 84 & 48 & 8 \\
\hline Aerosensitive C. jejuni $(n=20)$ & 100 & 95 & 50 & 75 & 50 & 65 & 45 & 5 \\
\hline Total $(n=70)$ & 100 & 97.1 & 68.6 & 81.4 & 57.1 & 84.3 & 64.3 & 11.4 \\
\hline
\end{tabular}

Statistical significance was performed by chi-square distribution with SPSS ver.21 (IBM). *P $\leq 0.05, * * P \leq 0.01, * * * * P \leq 0.0001$, NS, Not Significant; ND, Not determined.

impairs the internalization of C. jejuni into INT407 cells (Konkel et al., 1999b). The invasion-associated marker (iam) locus was first reported by Carvalho et al. (2001) with random amplified polymorphic DNA techniques (RAPD) and was detected in $85 \%$ of invasive strains and $20 \%$ of non-invasive strains. The detection frequencies of $p l d A$ were also significantly different between HAT and aerosensitive $C$. jejuni strains (Figure 2 and Table 2). The pldA gene encodes an outer membrane phospholipase A that is involved in hemolysis (Grant et al., 1997). The pldA and $c i a B$ genes also play a role in $C$. jejuni colonization of chicken intestines (Ziprin et al., 2001). The increased prevalence of the virulence genes in HAT C. jejuni strains suggests that HAT
C. jejuni would be more pathogenic to humans than aerosensitive C. jejuni.

The transmission of $C$. jejuni to humans is primarily mediated by contaminated food, mainly poultry meat. Due to the fastidiousness and oxygen sensitivity, C. jejuni is not expected to survive efficiently during foodborne transmission in oxygen-rich, atmospheric conditions. However, our results indicate that HAT C. jejuni survives longer in poultry meat than aerosensitive strains during transmission to humans in air and would be more capable of causing human infection (Figure 1). In this study, we observed that the survival of HAT C. jejuni is significantly reduced under $\mathrm{CO}_{2}$ (Figures 3,4 ). 

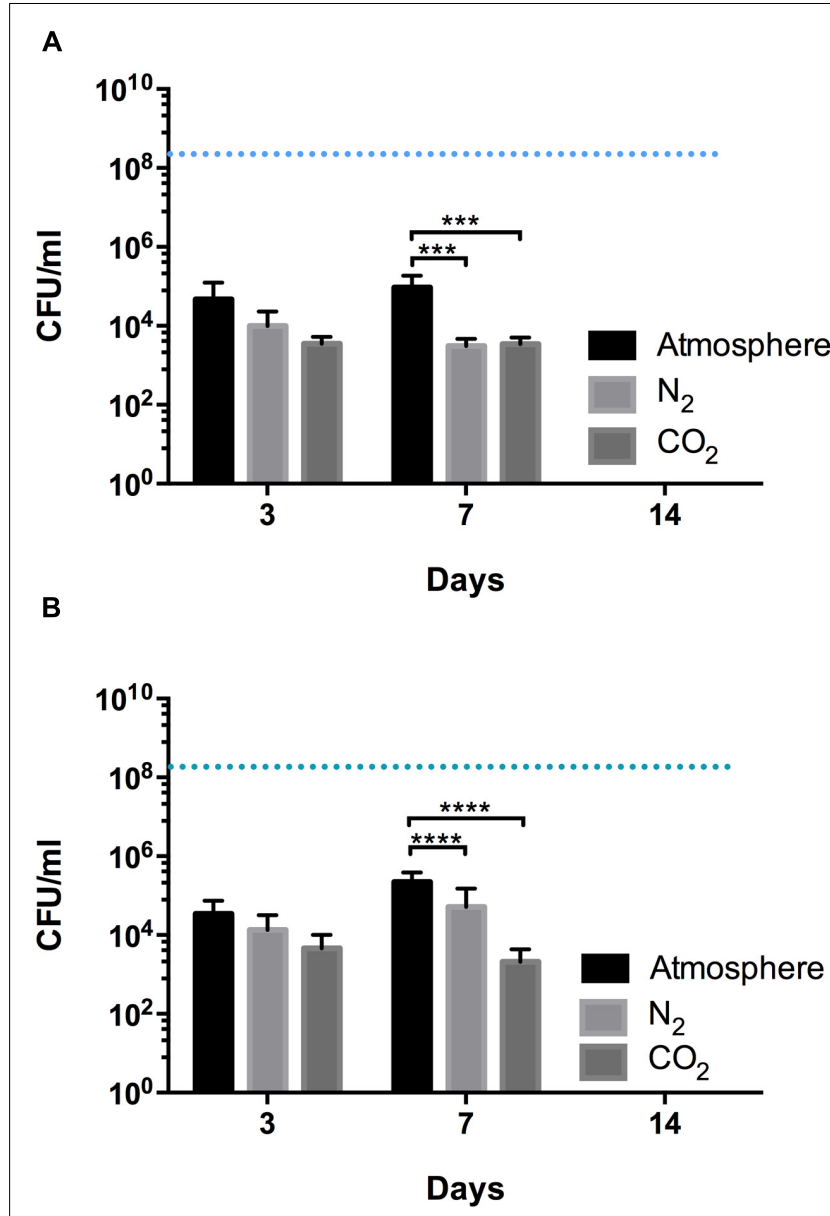

C

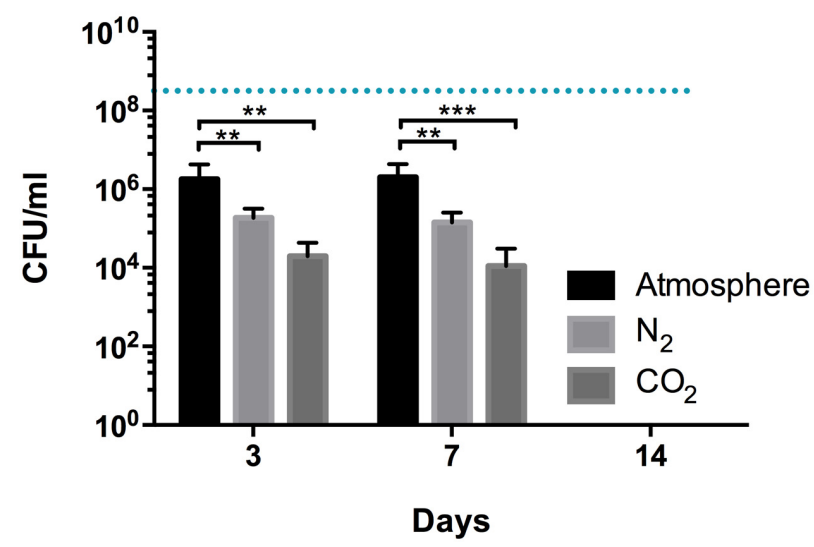

FIGURE 3 | Survival of aerosensitive (A), aerotolerant (B), and HAT (C) strains of $\mathrm{C}$. jejuni in $\mathrm{MH}$ broth under different gas conditions. Incubation was carried out in atmospheric, $\mathrm{N}_{2}$, and $\mathrm{CO}_{2}$ conditions. Two strains from each aerotolerance group were randomly selected, and each strain was inoculated in $\mathrm{MH}$ broth in triplicate. The initial CFU was adjusted to be approximately $10^{8}$ $\mathrm{CFU} / \mathrm{ml}$ for all the samples and is indicated with blue dashed lines. The results show the mean and standard deviation of the triplicate samples of two different strains in a single experiment. The experiment was repeated three times, and similar results were obtained in the three independent experiments. Two-way ANOVA testing was carried out for statistical analysis. ${ }^{* *} P \leq 0.01$, ${ }^{* * *} P \leq 0.001,{ }^{* * * *} P \leq 0.0001$.

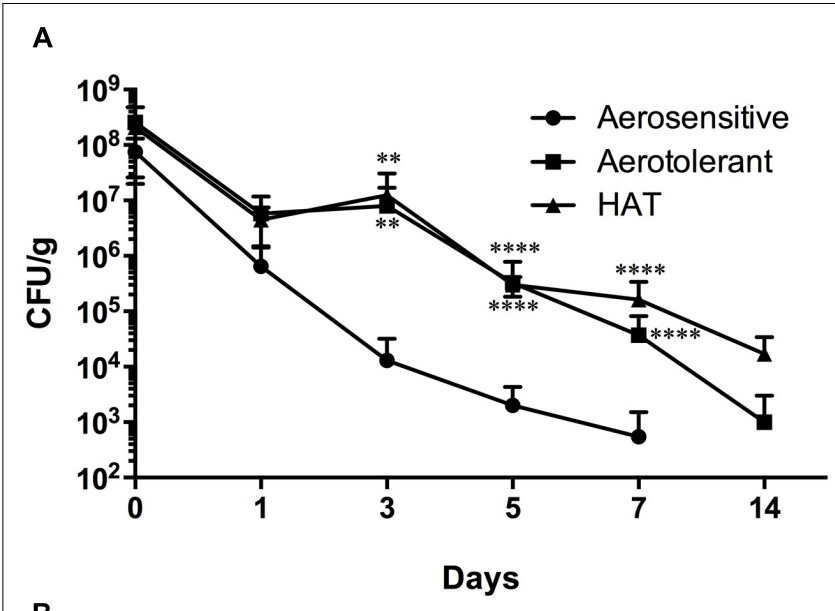

B

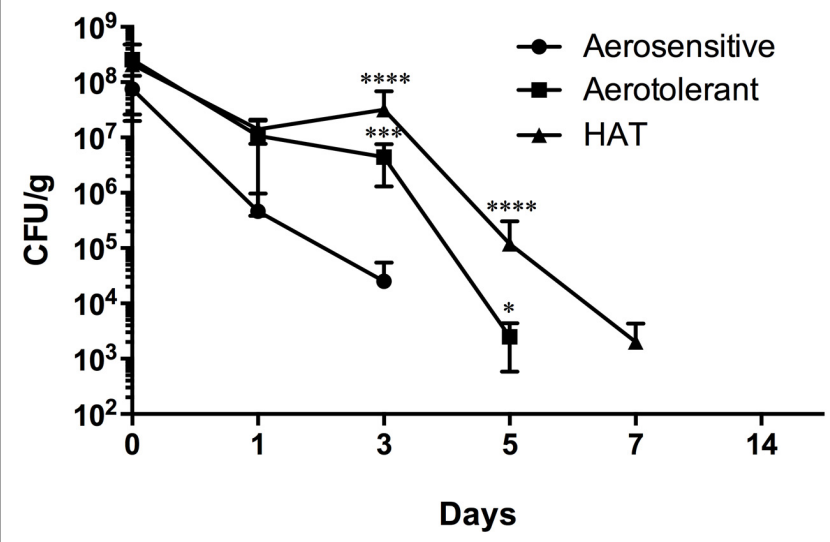

FIGURE 4 | Survival of aerosensitive, aerotolerant, and HAT C. jejuni strains at $4^{\circ} \mathrm{C}$ in poultry meat in $\mathrm{N}_{2}(\mathbf{A})$ and $\mathrm{CO}_{2}$ (B). Two strains from each aerotolerance group were randomly selected for the experiment. The results indicate the means and standard deviations of duplicate samples of the two different strains in a single experiment. Three independent experiments were performed, and similar results were obtained all the experiments. The statistical analysis was carried out with two-way ANOVA. ${ }^{*} P \leq 0.05$, ${ }^{* *} P \leq 0.01,{ }^{* * *} P \leq 0.001,{ }^{* * * *} P \leq 0.0001$.

This provides important scientific background for developing methods to control HAT C. jejuni with MAP. In food industry, $\mathrm{CO}_{2}, \mathrm{~N}_{2}$ and their combinations are generally used for the development of MAP of foods. Compared to aerobic conditions, the survival of HAT C. jejuni strains in raw poultry meat was significantly reduced by $\mathrm{CO}_{2}$ (Figures $\mathbf{1}$, 4B). Meredith et al. (2014) tested different compositions of the three gases and reported that 40:30:30 of $\mathrm{CO}_{2}: \mathrm{O}_{2}: \mathrm{N}_{2}$ is the optimum gas mixture both to reduce Campylobacter and to extend shelf-life in poultry filets. The threshold $\mathrm{CO}_{2}$ concentration that critically affects the viability of HAT C. jejuni has not been examined, and its determination still awaits future studies for the development of optimal gas mixtures of MAP to control HAT C. jejuni in poultry meat.

Our previous study revealed that most HAT C. jejuni strains belong to MLST CC 21 (Oh et al., 2015a), the major MLST CC 
implicated in human gastroenteritis (Nielsen et al., 2010). It is possible that strains of $C$. jejuni with increased aerotolerance may survive well in foods and are more likely to reach humans, consequently causing human illnesses more frequently than aerosensitive $C$. jejuni strains. At this stage, it remains unknown why HAT C. jejuni strains harbor more virulence genes than oxygen-sensitive strains. In this study, we did not provide empirical evidences about the virulence, such as invasion of and adhesion to epithelial cells, and such works will be done in future studies. Nevertheless, this study also showed that MAP using $\mathrm{CO}_{2}$ may be an interesting approach to control HAT C. jejuni in poultry meat.

\section{REFERENCES}

Bacon, D. J., Alm, R. A., Hu, L., Hickey, T. E., Ewing, C. P., Batchelor, R. A., et al. (2002). DNA sequence and mutational analyses of the pVir plasmid of Campylobacter jejuni 81-176. Infect. Immun. 70, 6242-6250. doi: 10.1128/IAI. 70.11.6242-6250.2002

Bang, D. D., Scheutz, F., Ahrens, P., Pedersen, K., Blom, J., and Madsen, M. (2001). Prevalence of cytolethal distending toxin $(c d t)$ genes and CDT production in Campylobacter spp. isolated from Danish broilers. J. Med. Microbiol. 50, 1087-1094. doi: 10.1099/0022-1317-50-12-1087

Biswas, D., Hannon, S. J., Townsend, H. G., Potter, A., and Allan, B. J. (2011). Genes coding for virulence determinants of Campylobacter jejuni in human clinical and cattle isolates from Alberta, Canada, and their potential role in colonization of poultry. Int. Microbiol. 14, 25-32.

Bolton, D. J. (2015). Campylobacter virulence and survival factors. Food Microbiol. 48, 99-108. doi: 10.1016/j.fm.2014.11.017

Bolton, F. J., and Coates, D. (1983). A study of the oxygen and carbon dioxide requirements of thermophilic campylobacters. J. Clin. Pathol. 36, 829-834. doi: $10.1136 /$ jcp.36.7.829

Bronowski, C., James, C. E., and Winstanley, C. (2014). Role of environmental survival in transmission of Campylobacter jejuni. FEMS Microbiol. Lett. 356, 8-19. doi: 10.1111/1574-6968.12488

Carvalho, A. C., Ruiz-Palacios, G. M., Ramos-Cervantes, P., Cervantes, L. E., Jiang, X., and Pickering, L. K. (2001). Molecular characterization of invasive and noninvasive Campylobacter jejuni and Campylobacter coli isolates. J. Clin. Microbiol. 39, 1353-1359. doi: 10.1128/JCM.39.4.1353-1359.2001

Chai, L.-C., Lee, H.-Y., Ghazali, F. M., Bakar, F. A., Malakar, P. K., Nishibuchi, M., et al. (2008). Simulation of cross-contamination and decontamination of Campylobacter jejuni during handling of contaminated raw vegetables in a domestic kitchen. J. Food Prot. 71, 2448-2452. doi: 10.4315/0362-028X-71.12. 2448

Datta, S., Niwa, H., and Itoh, K. (2003). Prevalence of 11 pathogenic genes of Campylobacter jejuni by PCR in strains isolated from humans, poultry meat and broiler and bovine faeces. J. Med. Microbiol. 52, 345-348. doi: 10.1099/jmm.0. 05056-0

Grant, K. A., Belandia, I. U., Dekker, N., Richardson, P. T., and Park, S. F. (1997). Molecular characterization of $p l d A$, the structural gene for a phospholipase A from Campylobacter coli, and its contribution to cell-associated hemolysis. Infect. Immun. 65, 1172-1180.

Hanning, I., Biswas, D., Herrera, P., Roesler, M., and Ricke, S. C. (2010). Prevalence and characterization of Campylobacter jejuni isolated from pasture flock poultry. J. Food Sci. 75, M496-M502. doi: 10.1111/j.1750-3841.2010.01747.x

Hermans, D., Van Deun, K., Messens, W., Martel, A., Van Immerseel, F., Haesebrouck, F., et al. (2011). Campylobacter control in poultry by current intervention measures ineffective: urgent need for intensified fundamental research. Vet. Microbiol. 152, 219-228. doi: 10.1016/j.vetmic.2011. 03.010

Jokinen, C., Edge, T. A., Ho, S., Koning, W., Laing, C., Mauro, W., et al. (2011). Molecular subtypes of Campylobacter spp., Salmonella enterica, and Escherichia coli $\mathrm{O} 157: \mathrm{H} 7$ isolated from faecal and surface water samples in the Oldman River watershed, Alberta, Canada. Water Res. 45, 1247-1257. doi: 10.1016/j. watres.2010.10.001

\section{AUTHOR CONTRIBUTIONS}

Design of the project: EO and BJ. Performance of the experiments: EO. Data analysis: EO, LM, LC, and BJ. Writing of the manuscript: EO, LM, LC, and BJ.

\section{ACKNOWLEDGMENTS}

This research was supported by funding from Alberta Livestock and Meat Agency, and the laboratory facilities were supported by the Canada Foundation for Innovation (CFI).

Kirk, M. D., Pires, S. M., Black, R. E., Caipo, M., Crump, J. A., Devleesschauwer, B., et al. (2015). World Health Organization estimates of the global and regional disease burden of 22 foodborne bacterial, protozoal, and viral diseases, 2010: a data synthesis. PLoS Med. 12:e1001921. doi: 10.1371/journal.pmed.1001921

Konkel, M. E., Gray, S. A., Kim, B. J., Garvis, S. G., and Yoon, J. (1999a). Identification of the enteropathogens Campylobacter jejuni and Campylobacter coli based on the cadF virulence gene and its product. J. Clin. Microbiol. 37, 510-517.

Konkel, M. E., Kim, B. J., Rivera-Amill, V., and Garvis, S. G. (1999b). Bacterial secreted proteins are required for the internaliztion of Campylobacter jejuni into cultured mammalian cells. Mol. Microbiol. 32, 691-701.

Leach, S., Harvey, P., and Wali, R. (1997). Changes with growth rate in the membrane lipid composition of and amino acid utilization by continuous cultures of Campylobacter jejuni. J. Appl. Microbiol. 82, 631-640. doi: 10.1111/j. 1365-2672.1997.tb02873.x

Luber, P. (2009). Cross-contamination versus undercooking of poultry meat or eggs - which risks need to be managed first? Int. J. Food Microbiol. 134, 21-28. doi: 10.1016/j.ijfoodmicro.2009.02.012

Melo, R. T., Nalevaiko, P. C., Mendonca, E. P., Borges, L. W., Fonseca, B. B., Beletti, M. E., et al. (2013). Campylobacter jejuni strains isolated from chicken meat harbour several virulence factors and represent a potential risk to humans. Food Control 33, 227-231. doi: 10.1016/j.foodcont.2013.02.032

Meredith, H., Valdramidis, V., Rotabakk, B. T., Sivertsvik, M., Mcdowell, D., and Bolton, D. J. (2014). Effect of different modified atmospheric packaging (MAP) gaseous combinations on Campylobacter and the shelf-life of chilled poultry fillets. Food Microbiol. 44, 196-203. doi: 10.1016/j.fm.2014.06.005

Muller, J., Schulze, F., Muller, W., and Hanel, I. (2006). PCR detection of virulenceassociated genes in Campylobacter jejuni strains with differential ability to invade Caco-2 cells and to colonize the chick gut. Vet. Microbiol. 113, 123-129. doi: 10.1016/j.vetmic.2005.10.029

Murphy, C., Carroll, C., and Jordan, K. N. (2006). Environmental survival mechanisms of the foodborne pathogen Campylobacter jejuni. J. Appl. Microbiol. 100, 623-632. doi: 10.1111/j.1365-2672.2006.02903.x

Nielsen, L. N., Sheppard, S. K., Mccarthy, N. D., Maiden, M. C., Ingmer, H., and Krogfelt, K. A. (2010). MLST clustering of Campylobacter jejuni isolates from patients with gastroenteritis, reactive arthritis and Guillain-Barre syndrome. J. Appl. Microbiol. 108, 591-599. doi: 10.1111/j.1365-2672.2009.04444.x

Oh, E., Kim, J. C., and Jeon, B. (2016). Stimulation of biofilm formation by oxidative stress in Campylobacter jejuni under aerobic conditions. Virulence 7, 846-851. doi: 10.1080/21505594.2016.1197471

Oh, E., Mcmullen, L., and Jeon, B. (2015a). High prevalence of hyper-aerotolerant Campylobacter jejuni in retail poultry with potential implication in human infection. Front. Microbiol. 6:1263. doi: 10.3389/fmicb.2015.01263

Oh, E., Mcmullen, L., and Jeon, B. (2015b). Impact of oxidative stress defense on bacterial survival and morphological change in Campylobacter jejuni under aerobic conditions. Front. Microbiol. 6:295. doi: 10.3389/fmicb.2015.00295

Parkhill, J., Wren, B. W., Mungall, K., Ketley, J. M., Churcher, C., Basham, D., et al. (2000). The genome sequence of the food-borne pathogen Campylobacter jejuni reveals hypervariable sequences. Nature 403, 665-668. doi: 10.1038/35001088

Rodrigues, R. C., Pocheron, A. L., Hernould, M., Haddad, N., Tresse, O., and Cappelier, J. M. (2015). Description of Campylobacter jejuni Bf, an atypical aero-tolerant strain. Gut Pathog. 7, 30. doi: 10.1186/s13099-015-0077-x 
Rosenquist, H., Nielsen, N. L., Sommer, H. M., Norrung, B., and Christensen, B. B. (2003). Quantitative risk assessment of human campylobacteriosis associated with thermophilic Campylobacter species in chickens. Int. J. Food Microbiol. 83, 87-103. doi: 10.1016/S0168-1605(02) 00317-3

Rozynek, E., Dzierzanowska-Fangrat, K., Jozwiak, P., Popowski, J., Korsak, D., and Dzierzanowska, D. (2005). Prevalence of potential virulence markers in Polish Campylobacter jejuni and Campylobacter coli isolates obtained from hospitalized children and from chicken carcasses. J. Med. Microbiol. 54, 615-619. doi: 10.1099/jmm.0.45988-0

Skarp, C. P., Hanninen, M. L., and Rautelin, H. I. (2016). Campylobacteriosis: the role of poultry meat. Clin. Microbiol. Infect. 22, 103-109. doi: 10.1016/j.cmi. 2015.11.019

Stahl, M., Butcher, J., and Stintzi, A. (2012). Nutrient acquisition and metabolism by Campylobacter jejuni. Front. Cell. Infect. Microbiol. 2:5. doi: 10.3389/fcimb. 2012.00005

Thomas, M. T., Shepherd, M., Poole, R. K., Van Vliet, A. H., Kelly, D. J., and Pearson, B. M. (2011). Two respiratory enzyme systems in Campylobacter jejuni NCTC 11168 contribute to growth on L-lactate. Environ. Microbiol. 13, 48-61. doi: 10.1111/j.1462-2920.2010.02307.x

Tracz, D. M., Keelan, M., Ahmed-Bentley, J., Gibreel, A., KowalewskaGrochowska, K., and Taylor, D. E. (2005). pVir and bloody diarrhea in Campylobacter jejuni enteritis. Emerg. Infect. Dis. 11, 838-843. doi: 10.3201/ eid1106.041052
Umaraw, P., Prajapati, A., Verma, A. K., Pathak, V., and Singh, V. P. (2017) Control of Campylobacter in poultry industry from farm to poultry processing unit-a review. Crit. Rev. Food Sci. Nutr. 57, 659-665. doi: 10.1080/10408398. 2014.935847

Velayudhan, J., and Kelly, D. J. (2002). Analysis of gluconeogenic and anaplerotic enzymes in Campylobacter jejuni: an essential role for phosphoenolpyruvate carboxykinase. Microbiology 148, 685-694. doi: 10.1099/00221287-148-3-685

Young, K. T., Davis, L. M., and Dirita, V. J. (2007). Campylobacter jejuni: molecular biology and pathogenesis. Nat. Rev. Microbiol. 5, 665-679. doi: $10.1038 /$ nrmicro 1718

Ziprin, R. L., Young, C. R., Byrd, J. A., Stanker, L. H., Hume, M. E., Gray, S. A., et al. (2001). Role of Campylobacter jejuni potential virulence genes in cecal colonization. Avian Dis. 45, 549-557. doi: 10.2307/1592894

Conflict of Interest Statement: The authors declare that the research was conducted in the absence of any commercial or financial relationships that could be construed as a potential conflict of interest.

Copyright (c) 2017 Oh, McMullen, Chui and Jeon. This is an open-access article distributed under the terms of the Creative Commons Attribution License (CC BY).

The use, distribution or reproduction in other forums is permitted, provided the original author(s) or licensor are credited and that the original publication in this journal is cited, in accordance with accepted academic practice. No use, distribution or reproduction is permitted which does not comply with these terms. 